\title{
ON THE VALUES AT INTEGERS OF THE DEDEKIND ZETA FUNCTION OF A REAL QUADRATIC FIELD
}

\author{
DAVID KRAMER
}

\begin{abstract}
In 1976 Shintani gave a decomposition of the Dedekind zeta function, $\zeta_{K}(s)$, of a totally real number field into a finite sum of functions, each given by a Dirichlet series whose meromorphic continuation assumes rational values at negative integers. He obtained a formula for these values, thereby giving an expression for $\zeta_{K}(-n), n=0,1,2, \ldots$ Earlier, Zagier had studied the special case of $\varsigma(A, s)$, the narrow ideal class zeta function for a real quadratic field. He decomposes $\zeta(A, s)$ into $\sum_{A} Z_{Q}(s)$, where $Z_{Q}(s)$ is given as a Dirichlet series associated to a binary quadratic form $Q(x, y)=a x^{2}+b x y+c y^{2}$, and the summation is over a canonically given finite cycle of "reduced" quadratic forms associated to a narrow ideal class $A$. He then obtains a formula for $Z_{Q}(-n)$ as a rational function in the coefficients of the form $Q$.

Since the denominator of $\varsigma(A,-n)$ is known not to depend on the class $A$, whereas the coefficients of reduced forms attain arbitrarily large values, it is natural to ask whether the rational function in Zagier's formula might be replaced by a polynomial. In this paper such a result is obtained. For example, Zagier gives
\end{abstract}

$$
\begin{aligned}
& 15120 \zeta(A,-2) \\
& =\sum_{A} \frac{b^{5}-10 a b^{3} c+30 a^{2} b c^{2}}{a^{3}}+\frac{b^{5}-10 a b^{3} c+30 a^{2} b c^{2}}{c^{3}}-21 b(a+c)
\end{aligned}
$$

while our result is

$15120 \zeta(A,-2)=\frac{1}{2}\left(\sum_{A}-\sum_{\theta A}\right)\left(60 a^{2}-117 a b+76 a c+38 b^{2}-117 b c+60 c^{2}\right)$, where $\theta$ is the narrow ideal class consisting of principal ideals generated by elements of negative norm.

Starting with a representation of $Z_{Q}(1+n)$ due to Shanks and Zagier for $n=1,2,3, \ldots$ as a certain transcendental function of the coefficients of $Q$, we also obtain the result that $\zeta(A, 1+n)$ is given as the same sum of reduced quadratic forms as in the formula for $\varsigma(A,-n)$, times the appropriate "gamma factor." This gives a new proof of the functional equation of $\zeta(A, s)$ at integer values of $s$, and suggests the possibility that one might be able to prove the functional equation for all $s$ by finding some relation between $Z_{Q}(s)$ and $Z_{Q}(1-s)$. So far we have not found such a relation.

Introduction. In 1976 Shintani [5] gave a decomposition of the Dedekind zeta function, $\varsigma_{K}(s)$, of a totally real number field into a finite sum of functions, each given by a Dirichlet series whose meromorphic continuation assumes rational values at negative integers. He obtained a formula for these values, thereby giving an expression for $\zeta_{K}(-n), n=0,1,2, \ldots$ Earlier, Zagier $[\mathbf{3}, 4]$ had studied the special

Received by the editors August 9, 1985.

1980 Mathematics Subject Classification (1985 Revision). Primary 11R42, 11M41; Secondary 11E32. 
case of $\zeta(A, s)$, the narrow ideal class zeta function for a real quadratic field. He decomposes $\zeta(A, s)$ into $\sum_{A} Z_{Q}(s)$, where $Z_{Q}(s)$ is given as a Dirichlet series associated to a binary quadratic form $Q(x, y)=a x^{2}+b x y+c y^{2}$, and the summation is over a canonically given finite cycle of "reduced" quadratic forms associated to the narrow ideal class $A$. He then obtains a formula for $Z_{Q}(-n)$ as a rational function in the coefficients of the form $Q$.

Since the denominator of $\varsigma(A,-n)$ is known not to depend on the class $A$, whereas the coefficients of reduced forms attain arbitrarily large values, it is natural to ask whether the rational function in Zagier's formula might be replaced by a polynomial. In this paper such a result is obtained. For example, Zagier gives

$$
15120 \varsigma(A,-2)=\sum_{A} \frac{b^{5}-10 a b^{3} c+30 a^{2} b c^{2}}{a^{3}}+\frac{b^{5}-10 a b^{3} c+30 a^{2} b c^{2}}{c^{3}}-21 b(a+c)
$$

while our result is

$$
15120 \zeta(A,-2)=\frac{1}{2}\left(\sum_{A}-\sum_{\theta A}\right)\left(60 a^{2}-117 a b+76 a c+38 b^{2}-117 b c+60 c^{2}\right),
$$

where $\theta$ is the narrow ideal class consisting of principal ideals generated by elements of negative norm.

Starting with a representation of $Z_{Q}(1+n)$ due to Shanks and Zagier [2] for $n=1,2,3, \ldots$ as a certain transcendental function of the coefficients of $Q$, we also obtain the result that $\zeta(A, 1+n)$ is given as the same sum of reduced quadratic forms as in the formula for $\zeta(A,-n)$, time the appropriate "gamma factor." This gives a new proof of the functional equation of $\zeta(A, s)$ of integer values of $s$, and suggests the possibility that one might be able to prove the functional equation for all $s$ by finding some relation between $Z_{Q}(s)$ and $Z_{Q}(1-s)$. So far we have not found such a relation.

1. Binary quadratic forms. Unless otherwise indicated, $D$ will always denote a positive integer, not a perfect square. Let $Q(x, y)=a x^{2}+b x y+c y^{2}$ be a binary quadratic form with integral coefficients, and of discriminant $D=b^{2}-4 a c$. We shall freely use $Q$ or $(a, b, c)$ to denote this form. Unless otherwise noted, $Q$ will always be understood to be primitive; that is, g.c.d. $(a, b, c)=1$. We shall also allow ourselves a small degree of sloppiness and write "quadratic form" or jut "form," but shall always mean "binary quadratic form."

Recall that $\mathrm{GL}_{2}(\mathbf{Z})$ acts on the collection of forms of discriminant $D$ as follows: for $T=\left(\begin{array}{ll}\alpha & \beta \\ \gamma & \delta\end{array}\right), Q \mid T=: Q\left((x, y)^{t} T\right)=\mu Q(\alpha x+\beta y, \gamma x+\delta y)$, where $\mu=\alpha \delta-\beta \gamma=$ \pm 1 .

Under this action we define an equivalence of quadratic forms.

DEFINITION 1. Two forms $Q_{1}$ and $Q_{2}$ are equivalent in the narrow sense if $Q_{1}=Q_{2} \mid T$ for some $T \in \mathrm{SL}_{2}(\mathbf{Z}) . Q_{1}$ is equivalent to $Q_{2}$ in the wide sense if $Q_{1}=Q_{2} \mid T$ for some $T \in \mathrm{GL}_{2}(\mathbf{Z})$.

PROPOSITION 1. (i) The action of $\mathrm{SL}_{2}(\mathbf{Z})$ (resp. $\mathrm{GL}_{2}(\mathbf{Z})$ ) on the collection of forms of discriminant $D$ yields a finite number of equivalence classes. This number is called the narrow (resp. wide) class number of $D$, and is denoted $h(D)$ (resp. $\left.h_{0}(D)\right)$. 
(ii) For a given $D$, either the notions of narrow and wide equivalence coincide, in which case $h(D)=h_{0}(D)$, or else each wide class is composed of two distinct narrow classes, and $h(D)=2 h_{0}(D)$. (See, for instance, $[\mathbf{1}$, Chapter 2].)

DEFINITION 2. Let $A$ be a narrow equivalence class of quadratic forms of discriminant $D$. We define the following classes, associated to $A$ :

$$
\begin{aligned}
& \theta A=\left\{Q(x, y) \mid T ; Q \in A \text { and } T \in \mathrm{GL}_{2}(\mathbf{Z})-\mathrm{SL}_{2}(\mathbf{Z})\right\} \\
& A^{\prime}=\left\{-Q(x, y) \mid\left(\begin{array}{ll}
0 & 1 \\
1 & 0
\end{array}\right), Q \in A\right\}=\{Q(y, x) \mid Q(x, y) \in A\}
\end{aligned}
$$

Proposition 2. Let $A$ be a narrow equivalence class of quadratic forms. If the form $(a, b, c) \in A$, then

(i) $(c, b, a) \in A^{\prime}$,

(ii) $(-c,-b,-a) \in \theta A$,

(iii) $(a,-b, c) \in A^{\prime}$,

(iv) $(-a,-b,-c) \in \theta A^{\prime}$.

DEFINITION 3. A binary quadratic form $(a, b, c)$ is called reduced (in the narrow sense) if $a>0, c>0, b>a+c$.

The following proposition is proved by Zagier in [3].

Proposition 3. Let $A$ be a narrow equivalence class of forms, and $\theta A$ the "adjoint" class as in Definition 2. Let $F(x, y)$ be a function of two variables satisfying $F(x, y)= \pm F(1 / x, 1 / y)$. Then

$$
\left(\sum_{A} \pm \sum_{\theta A}\right)\left(F\left(w-1,1-w^{\prime}\right)-F\left(1-1 / w, 1 / w^{\prime}-1\right)\right)=0
$$

where the summation is over all reduced forms $(a, b, c)$ in the given class, with $w=(b+\sqrt{D}) / 2 a, w^{\prime}=(b-\sqrt{D}) / 2 a$.

Since there are only finitely many reduced forms of a given discriminant, this sum is well defined. Zagier's proof of Proposition 3 relies on the representation of $w$ as a purely periodic "-" continued fraction. We shall restate the proposition in terms of the coefficients themselves, and offer our own proof, which avoids the use of continued fractions. Observe that if $w$ and $w^{\prime}$ as above correspond to the form $(a, b, c)$, then $\left(1 / w, 1 / w^{\prime}\right)$ corresponds to $(-c,-b,-a) ;\left(w-1, w^{\prime}-1\right)$ to $(a, b-2 a, a-b+c)$; and $\left(1-1 / w, 1-1 / w^{\prime}\right)$ to the form $(c, 2 c-b, c-b+a)$, all forms of a given fixed discriminant $D$. Thus, Proposition 3 may be restated as

Proposition $3^{\prime}$. Let $A$ be a narrow equivalence class of forms, and $\theta A$ the adjoint class. Let $F(Q)$ be a function on binary quadratic forms satisfying $F(a, b, c)=$ $\pm F(-c,-b,-a)$. Then

$$
\left(\sum_{A} \pm \sum_{\theta A}\right)(F(a, b-2 a, a-b+c)-F(c, 2 c-b, c-b+a))=0 .
$$


PROOF. For the proof we need the following

LEMMA 1. The set of forms $\left(a^{\prime}, b^{\prime}, c^{\prime}\right) \in A$ with $a^{\prime}>0>c^{\prime}$ is the disjoint union of the sets of forms

(1) $(a, b-2 a, a-b+c)$ with $(a, b, c) \in A,(a, b, c)$ reduced,

(2) $(-c+b-a, 2 a-b,-a)$ with $(a, b, c) \in \theta A,(a, b, c)$ reduced.

ProOF. If $(a, b-2 a, a-b+c)=(-C+B-A, 2 A-B,-A)$, a simple calculation shows that $c=-C$, so $(a, b, c)$ and $(A, B, C)$ cannot both be reduced. Thus sets (1) and (2) are disjoint.

The form $(a, b-2 a, a-b+c)=(a, b, c) \mid\left(\begin{array}{cc}1 & -1 \\ 0 & 1\end{array}\right)$, and since $\left(\begin{array}{cc}1 & -1 \\ 0 & 1\end{array}\right) \in \mathrm{SL}_{2}(\mathbf{Z})$, $(a, b, c) \in A$ implies $(a, b-2 a, a-b+c) \in A$. Moreover, the form $(-c+b-a, 2 a-b,-a)$ is equal to $(a, b, c) \mid\left(\begin{array}{cc}1 & -1 \\ -1 & 0\end{array}\right)$, and since $\left(\begin{array}{cc}1 & -1 \\ -1 & 0\end{array}\right) \in \mathrm{GL}_{2}(\mathbf{Z})-\mathrm{SL}_{2}(\mathbf{Z})$, it follows that $(a, b, c) \in \theta A$ implies $(-c+b-a, 2 a-b,-a) \in A$.

Conversely, suppose that $Q=\left(a^{\prime}, b^{\prime}, c^{\prime}\right) \in A$ with $a^{\prime}>0>c^{\prime}$. We must show that $Q$ belongs to set (1) or (2). Let $Q_{1}=\left(a^{\prime}, b^{\prime}+2 a^{\prime}, a^{\prime}+b^{\prime}+c^{\prime}\right)$ and $Q_{2}=\left(-c^{\prime},-b^{\prime}-2 c^{\prime},-a^{\prime}-b^{\prime}-c^{\prime}\right)$. We claim that precisely one of these forms is reduced. Clearly at most one of them is reduced, since the third components have opposite signs. If $a^{\prime}+b^{\prime}+c^{\prime}>0$, then, since $a^{\prime}>0>c^{\prime}$, it follows that $b^{\prime}+2 a^{\prime}>$ $a^{\prime}+b^{\prime}+c^{\prime}>0$, and hence $Q_{1}$ is reduced. Since $\left(a^{\prime}, b^{\prime}, c^{\prime}\right)=Q_{1} \mid\left(\begin{array}{cc}1 & -1 \\ 0 & 1\end{array}\right),\left(a^{\prime}, b^{\prime}, c^{\prime}\right)$ belongs to set (1). Similarly, if $a^{\prime}+b^{\prime}+c^{\prime}<0$, then $Q_{2}$ is reduced, and since $\left(a^{\prime}, b^{\prime}, c^{\prime}\right)=Q_{2} \mid\left(\begin{array}{cc}1 & -1 \\ -1 & 0\end{array}\right)$ it follows that $\left(a^{\prime}, b^{\prime}, c^{\prime}\right)$ is in set $(2)$.

REMARK. We can also express the set of $\left(a^{\prime}, b^{\prime}, c^{\prime}\right) \in A$ with $a^{\prime}>0>c^{\prime}$ as the disjoint union of

(3) $(c, 2 c-b, a-b+c) ;(a, b, c) \in A$, reduced,

(4) $(-a+b-c, b-2 c,-c) ;(a, b, c) \in \theta A$, reduced.

The proof is entirely analogous to that of Lemma 1.

The proof of the proposition is now straightforward:

$F(a, b, c)= \pm F(-c,-b,-a)$ implies that

$$
\begin{aligned}
\left(\sum_{A}\right. & \left. \pm \sum_{\theta A}\right)(F(a, b-2 a, a-b+c)-F(c, 2 c-b, a-b+c)) \\
= & \sum_{A}(F(a, b-2 a, a-b+c)-F(c, 2 c-b, c-b+a)) \\
& +\sum_{\theta A}(F(-a+b-c, 2 a-b,-a)-F(-a+b-c, b-2 c,-c))
\end{aligned}
$$

which by Lemma 1 and the remark equals

$$
\sum_{a>0>c} F(a, b, c)-\sum_{a>0>c} F(a, b, c)=0 .
$$

2. The correspondence between equivalence classes of forms of positive discriminant and module classes in real quadratic fields. There is a natural correspondence between equivalence classes of modules and $\mathrm{SL}_{2}(\mathbf{Z})$-equivalence classes of primitive binary quadratic forms. Namely, to a basis $\xi_{1}, \xi_{2}$ of $M$ we associate the form $Q(x, y)=N\left(x \xi_{1}+y \xi_{2}\right) / N(M)$, where $N$ is the norm. Then $Q$ is a primitive form of discriminant $D$ with rational integral coefficients. If we consider only oriented bases for $M,\left(\xi_{1} \xi_{2}^{\prime}-\xi_{1}^{\prime} \xi_{2}>0\right)$, then the $\mathrm{SL}_{2}(\mathbf{Z})$-equivalence class 
of forms to which $Q$ belongs is associated to the collection of ordered bases of $M$. Moreover, $Q$ remains unchanged if $M$ is replaced by $\lambda M(\lambda \gg 0)$. Thus, we have a bijection between equivalence classes of modules $M$ for which $O_{M}$, the multiplier ring of $M$, has discriminant $D$, and $\mathrm{SL}_{2}(\mathbf{Z})$-equivalence classes of primitive binary quadratic forms of discriminant $D$. For details, see, for example, [4 or 1].

3. Module class zeta functions of a real quadratic field. Let $M$ be a module of rank 2 in a real quadratic field. The zeta function of $M$ is defined by

(1) $\varsigma(M, s)=N(M)^{s} \sum_{\substack{\xi \in M / E \\ \xi \gg 0}} \frac{1}{N(\xi)^{s}} ; \quad \begin{array}{r}\mathrm{re}(s)>1, E \text { the group of totally } \\ \text { positive units } \varepsilon \text { satisfying } \varepsilon M=M .\end{array}$

Since $\zeta(M, s)=\varsigma(\lambda M, s)$ for $\lambda \gg 0, \zeta(M, s)$ can be written as $\zeta(B, s)$, the zeta function of $B$, the module class to which $M$ belongs. In [3 and 4] Zagier proves the following theorems.

Let $Q(p, q)=\left(a p^{2}+b p q+c q^{2}\right)$ be a quadratic form of discriminant $D>0$ with positive coefficients. Define

$$
Z_{Q}(s)=\sum_{p, q}^{\prime} \frac{1}{Q(p, q)^{s}} ; \quad \operatorname{re}(s)>1,
$$

where

$$
\sum_{p, q}{ }^{\prime} f(p, q)=\sum_{p=1}^{\infty} \sum_{q=1}^{\infty} f(p, q)+\frac{1}{2} \sum_{p=1}^{\infty} f(p, 0)+\frac{1}{2} \sum_{q=1}^{\infty} f(0, q) .
$$

THEOREM 1. $\varsigma(B, s)=\sum_{Q} Z_{Q}(s)$, where the summation is over the reduced forms in the class of forms associated to the module class of $M$.

THEOREM 2. The function $Z_{Q}(s)$ has a meromorphic continuation to all of $\mathbf{C}$, holomorphic except for a pole at $s=1$. The values of $Z_{Q}(s)$ at nonpositive integers can be expressed in terms of rational functions in $a, b, c$ with rational coefficients. To be precise, for $Q=(a, b, c)$, define rational functions in $a, b, c$ for $k=0,1,2, \ldots$ by

$$
Z_{k}(Q)=\frac{(-1)^{k} k !}{2} \frac{B_{2 k+2}}{2 k+2}\left(\frac{f_{k}(a, b, c)}{a^{k+1}}+\frac{f_{k}(a, b, c)}{c^{k+1}}\right)+g_{k}(a,-b, c)
$$

for $k \geq 0$, where $B_{n}$ is the nth Bernoulli number, and $f_{k}$ and $g_{k}$ are polynomials defined by

$$
\begin{gathered}
f_{k}(a, b, c)=\sum_{r=0}^{k} \frac{(-1)^{r}(k-r) !}{r !(2 k+1-2 r) !} a^{r} b^{2 k+1-r} c^{r}, \\
g_{k}(a, b, c)=-\sum_{r=0}^{2 k} \frac{B_{r+1}}{r+1} \frac{B_{2 k+1-r}}{2 k+1-r} d_{r, k+1}(a, b, c),
\end{gathered}
$$

where $d_{r, k}$ is defined by

$$
\sum_{r=0}^{2 k-2} d_{r, k}(a, b, c) x^{r} y^{2 k-2-r}=\left(a x^{2}+b x y+c y^{2}\right)^{k-1}
$$


Then

$$
Z_{Q}(-k)=Z_{k}(Q)
$$

In particular,

$$
\begin{aligned}
Z_{Q}(0)= & \frac{1}{24}\left(\frac{b}{a}+\frac{b}{c}\right)-\frac{1}{4} \\
Z_{Q}(-1)= & \frac{1}{1440}\left(\frac{b^{3}-6 a b c}{a^{2}}+\frac{b^{3}-6 a b c}{c^{2}}\right)+\frac{1}{144} b \\
Z_{Q}(-2)= & \frac{1}{15120}\left(\frac{b^{5}-10 a b^{3} c+30 a^{2} b c^{2}}{a^{3}}+\frac{b^{5}-10 a b^{3} c+30 a^{2} b c^{2}}{c^{3}}\right) \\
& +\frac{1}{720} b(a+c) .
\end{aligned}
$$

(The formula for $Z_{Q}(-2)$ is given incorrectly in [4].) Combining Theorems 1 and 2 gives an explicit formula for $\varsigma(B,-k)$.

It is known that the denominator of $\zeta(B,-k)$, when expressed in lowest terms, has a bound independent of the discriminant and of the choice of module class. The existence of such a bound is not, however, indicated by Zagier's formula, since the coefficients of the reduced forms, which can be arbitrarily large, appear in the denominator of $Z_{n}(Q)$. Zagier remedies this situation, though at the cost of introducing the transition numbers of the continued fraction for $w=(b+\sqrt{D}) / 2 a$, as follows: Let $Q$ be a reduced form with root

$$
w=\left(\left(b_{0}, b_{1}, \ldots, b_{t}\right)\right)=b_{0}-\frac{1}{b_{1}}-\frac{1}{b_{2}}-\cdots
$$

Then Zagier proves the following result:

THEOREM 3.

$$
\begin{array}{r}
\varsigma(B,-k)=\sum_{n=1}^{t} \sum_{s=0}^{2 k}\left(\frac{B_{2 k+2}}{2 k+2} \frac{b_{n} 2 k-s+1}{2 k-s+1}-\frac{B_{s+1}}{s+1} \frac{B_{2 k-s+1}}{2 k-s+1}\right) d_{s, k+1}\left(Q_{n}\right), \\
k=0,1,2, \ldots,
\end{array}
$$

where $Q_{n}$ is the form associated to the $n$th cyclic permutation of $\left(\left(b_{0}, b_{1}, \ldots, b_{t}\right)\right)$.

In this formula the only contribution to the denominator comes from Bernoulli numbers, which depend only on $k$.

DEFINITION 4 . Let $B$ be a narrow equivalence class of quadratic forms. We define

$$
\varsigma_{+}(B, s)=\varsigma(B, s)+\varsigma(\theta B, s), \quad \varsigma_{-}(B, s)=\varsigma(B, s)-\varsigma(\theta B, s) .
$$

Our first main result is a formula for $\varsigma(B,-k)$ which is the sum over the reduced forms of both $B$ and $\theta B$, of a certain polynomial in the coefficients of $Q$. Thus we obtain a universal bound on the denominator of $\zeta(B,-k)$ without introducing the $b_{n}$ of Theorem 3; however, we are compelled to sum over the forms of both $B$ and $\theta B$. 
We first define two equivalence relations on the set of functions of binary quadratic forms.

DEFINITION 5. A function on quadratic forms $\Phi=\Phi(Q)=\Phi\left(w, w^{\prime}\right)$ will be said to be "+"-equivalent to 0 , written $\Phi^{+} 0$, if there is a function $F$ satisfying $F\left(w, w^{\prime}\right)=F\left(1 / w, 1 / w^{\prime}\right)$ for all forms, such that

$$
\Phi\left(w, w^{\prime}\right)=F\left(w-1,1-w^{\prime}\right)-F\left(1-1 / w, 1 / w^{\prime}-1\right) .
$$

$\Phi$ will be called "-"-equivalent to zero $(\Phi \approx 0)$ if

$$
\Phi\left(w, w^{\prime}\right)=F\left(w-1,1-w^{\prime}\right)-F\left(1-1 / w, 1 / w^{\prime}-1\right)
$$

for a function $F$ satisfying $F\left(w, w^{\prime}\right)=-F\left(1 / w, 1 / w^{\prime}\right)$. We further define $\Phi_{1} \stackrel{ \pm}{\sim} \Phi_{2}$ if $\left.\Phi_{1}-\Phi_{2} \stackrel{ \pm}{\sim} 0\right)$.

An immediate corollary to Proposition 3 is the following:

PROPOSITION 4. Let $B$ be a narrow equivalence class of forms and $\Phi$ a function on quadratic forms. Then if $\Phi \stackrel{ \pm}{\sim}$, it follows that $\left(\sum_{B} \pm \sum_{\theta B}\right) \Phi(Q)=0$, where summation is over the reduced forms of the indicated classes.

THEOREM 4. For a quadratic form $Q=(a, b, c)$ define the polynomials $K_{k}(Q)$, $k=1,2,3, \ldots$, by the following formula:

$$
\begin{aligned}
K_{k}(Q)= & -2 g_{k}(a, b, c)+g_{k}(a, b-2 a, a-b+c)+g_{k}(c, b-2 c, c-b+a) \\
& +B_{2 k+2} \sum_{n=0}^{2 k} \frac{d_{n, k+1}(a,-b, c)}{(n+1)(2 k+1-n)}
\end{aligned}
$$

with $g_{k}$ as in (5) and $d_{n, k}$ as in (6).

Then $Z_{Q}(-k)^{(-1)^{k+1}} K_{k}(Q)$, and hence

$$
\begin{aligned}
& \zeta_{+}(B,-k)=\left(\sum_{B}+\sum_{B}\right) K_{k}(Q) \text { for } k \text { odd }, \\
& \varsigma_{-}(B,-k)=\left(\sum_{B}-\sum_{\theta B}\right) K_{k}(Q) \text { for } k \text { even. }
\end{aligned}
$$

THEOREM 5. $Z_{Q}(-k) \stackrel{(-1)^{k}}{\sim} 0$.

COROLLARY. From Theorem 5 follows the well-known fact that $\zeta_{+}(B,-k)=0$ for $k$ even, and $\zeta_{-}(B,-k)=0$ for $k$ odd.

THEOREM 6. Let

$$
\delta_{k}=\delta_{k}(D)=\frac{2^{2 k} D^{-\left(k+\frac{1}{2}\right)} \pi^{2 k+2}}{\Gamma^{2}(k+1)}
$$

Then $Z_{Q}(1+k)^{(-1)^{k+1}} \delta_{k} K_{k}(Q)$.

Combining Theorems 4 and 6, we have the following

Corollary. $Z_{Q}(-k)^{(-1)^{k+1}} \delta_{k}^{-1} Z_{Q}(1+k)$. 
This, together with Theorem 5 , represents a refinement of the functional equations for $\zeta_{ \pm}(B, s)$ at integer values; namely,

(i) $\varsigma_{+}(B,-k)=\delta_{k}^{-1} \zeta_{+}(B, 1+k)$, and $\zeta_{-}(B,-k)=0$ for $k$ odd, $k>0$; while

(ii) $\zeta_{-}(B,-k)=\delta_{k}^{-1} \zeta_{-}(B, 1+k)$, and $\zeta_{+}(B,-k)=0$ for $k$ even, $k>0$.

We shall prove (ii) by showing that $\delta_{k}^{-1} Z_{Q}(1+k)^{(-1)^{k+1}} K_{k}(Q)$.

ProOF OF THEOREM 4. We must find functions $F_{k}(Q), k \geq 1$, satisfying

(i) $F_{k}\left(w, w^{\prime}\right)=(-1)^{k+1} F_{k}\left(1 / w, 1 / w^{\prime}\right)$.

(ii) $F_{k}\left(w-1,1-w^{\prime}\right)-F_{k}\left(1-1 / w, 1 / w^{\prime}-1\right)=Z_{k}(Q)-K_{k}(Q)$.

Let

$$
F_{k}(Q)=D^{k / 2} \frac{(-1)^{k} k !}{2} \frac{B_{2 k+2}}{2 k+2}\left(w+w^{\prime}\right)^{-k} S_{k}\left(w, w^{\prime}\right)
$$

where

$$
S_{k}\left(w, w^{\prime}\right)=f_{k}\left(w, w-w^{\prime},-w^{\prime}\right)+(-1)^{k+1}\left(w w^{\prime}\right)^{k} f_{k}\left(\frac{1}{w}, \frac{w^{\prime}-w}{w w^{\prime}}, \frac{-1}{w^{\prime}}\right),
$$

$f_{k}$ being given in (4).

We claim the desired properties (i) and (ii) for the $F_{k}$.

One observes by inspection that (i) is satisfied. To establish (ii) we define for a form $(a, b, c)$ of discriminant $D$, and for $k=1,2,3, \ldots$ the polynomials

$$
\varphi_{k}(Q)=\varphi_{k}(D, b)=\sum_{r=0}^{k} \frac{(k-r) !}{r !(2 k-2 r+1) !}\left(\frac{D-b^{2}}{4}\right)^{r} b^{2 k+1-2 r}
$$

(note: $\varphi_{k}\left(b^{2}-4 a c, b\right)=f_{k}(a, b, c)$ ).

Then, observing that $w-w^{\prime}=\sqrt{D} / a, w+w^{\prime}=b / a$, and $w w^{\prime}=c / a$, we have

$$
\begin{aligned}
F_{k}\left(w-1,1-w^{\prime}\right)=\frac{(-1)^{k} k !}{2} \frac{B_{2 k+2}}{2 k+2} \\
\cdot\left(a^{k} \varphi_{k}\left(\frac{D}{a^{2}}, \frac{b-2 a}{a}\right)+(a-b+c)^{k} \varphi_{k}\left(\frac{D}{(c-b+a)^{2}}, \frac{2 a-b}{a-b+c}\right)\right)
\end{aligned}
$$

and

$$
\begin{aligned}
F_{k}\left(1-\frac{1}{w},\right. & \left.\frac{1}{w^{\prime}}-1\right)=\frac{(-1)^{k+1} k !}{2} \frac{B_{2 k+2}}{2 k+2} \\
& \cdot\left(c^{k} \varphi_{k}\left(\frac{D}{c^{2}}, \frac{b-2 c}{c}\right)+(c-b+a)^{k} \varphi_{k}\left(\frac{D}{(c-b+a)^{2}}, \frac{2 c-b}{c-b+a}\right)\right) .
\end{aligned}
$$

We have, therefore,

$$
\begin{aligned}
& F_{k}\left(w-1,1-w^{\prime}\right)-F_{k}\left(1-\frac{1}{w}, \frac{1}{w^{\prime}}-1\right) \\
& 10) \quad-\frac{(-1)^{k} k !}{2} \frac{B_{2 k+2}}{2 k+2}\left(\frac{f_{k}(a, b, c)}{a^{k+1}}+\frac{f_{k}(a, b, c)}{c^{k+1}}\right) \\
& =\frac{(-1)^{k} k !}{2} \frac{B_{2 k+2}}{2 k+2}\left(\frac{\varphi_{k}(D, b-2 a)-\varphi_{k}(D, b)}{a^{k+1}}+\frac{\varphi_{k}(D, b-2 c)-\varphi_{k}(D, b)}{c^{k+1}}\right. \\
& \left.+\frac{\varphi_{k}(D, 2 a-b)-\varphi_{k}(D, b-2 c)}{(c-b+a)^{k+1}}\right),
\end{aligned}
$$


where we have used

$$
\varphi_{k}(D,-b)=-\varphi_{k}(D, b) \text { and } \varphi_{k}\left(\lambda^{2} D, \lambda b\right)=\lambda^{2 k+1} \varphi_{k}(D, b) .
$$

Now we employ the identity

$$
\begin{aligned}
& \frac{\varphi_{k}(D, x)-\varphi_{k}(D, x-2 y)}{y^{k+1}} \\
& =(-1)^{k} \frac{2}{k !} \sum_{r=0}^{2 k}\left(\frac{y}{\alpha}\right)^{k-r} \frac{1}{(2 k-r+1)} d_{r, k+1}\left(\frac{x^{2}-D}{4 \alpha},-x, \alpha\right)
\end{aligned}
$$

(Proposition 4 in [4]), as well as the observation that

$$
(a / c)^{k-r} d_{r, k}(a, b, c)=d_{r, k}(c, b, a) \quad \text { and } \quad d_{r, k}(a, b, c)=(-1)^{r} d_{r, k}(a,-b, c)
$$

to deduce that the right-hand side of $(10)$ is equal to

$$
-\frac{B_{2 k+2}}{2 k+2} \sum_{r=0}^{2 k}\left(\frac{d_{r, k+1}(c,-b, a)}{2 k+1-r}+\frac{d_{r, k+1}(a,-b, c)}{2 k+1-r}+(-1)^{r-1} \frac{d_{r, k(a, 2 a-b, a-b+c)}}{2 k+1-r}\right),
$$

where to obtain the last summand we apply (11) to the last summand in (10) with $x=2 a-b, y=a-b+c, \alpha=a$.

Collecting terms, and using the definitions of $Z_{k}(Q)$ and $K_{k}(Q)$, we may write our result so far as follows:

$$
F_{k}\left(w-1,1-w^{\prime}\right)-F_{k}\left(1-\frac{1}{w}, \frac{1}{w^{\prime}}-1\right)=Z_{k}(Q)-K_{k}(Q)+J_{k}(Q),
$$

where

$$
\begin{aligned}
J_{k}(Q)=\frac{B_{2 k+2}}{2 k+2} \sum_{n=0}^{2 k}\left(\frac{-d_{n, k+1}(c,-b, a)}{2 k+1-n}+\frac{d_{n, k+1}(a, b-2 a, c-b+a)}{2 k+1-n}\right. \\
\left.-\frac{d_{n, k+1}(a,-b, c)}{2 k+1-n}\right)
\end{aligned}
$$

$$
\begin{aligned}
& +B_{2 k+2} \sum_{n=0}^{2 k} \frac{1}{(n+1)(2 k+1-n)} d_{n, k+1}(a,-b, c) \\
& +\sum_{n=0}^{2 k} \frac{B_{n+1}}{n+1} \frac{B_{2 k+1-n}}{2 k+1-n}\left(d_{n, k+1}(a, 2 a-b, c-b+a)\right. \\
& \left.+d_{n, k+1}(c, 2 c-b, a-b+c)-d_{n, k+1}(a,-b, c)\right) .
\end{aligned}
$$

It now suffices to show that $J_{k}(Q)$ is identically zero.

LEMMA 2.

$$
d_{n, k}(a+b+c, b+2 c, c)=\sum_{j=0}^{n}\left(\begin{array}{c}
2 k-2-j \\
n-j
\end{array}\right) d_{j, k}(a, b, c) .
$$

ProOF. Let $P_{k}(x, y)=\left(a x^{2}+b x y+c y^{2}\right)^{k-1}$. Then

$$
d_{j, k}(a, b, c)=\frac{1}{2 \pi i} \oint\left(\frac{P_{k}(x, 1)}{x^{j+1}} d x\right) .
$$


Thus

$$
\begin{aligned}
& \sum_{j=0}^{n}\left(\begin{array}{c}
2 k-2-j \\
n-j
\end{array}\right) d_{j, k}(a, b, c)=\sum_{j \in \mathbf{Z}}\left(\begin{array}{c}
2 k-2-j \\
n-j
\end{array}\right) d_{j, k}(a, b, c) \\
& \quad=\frac{1}{2 \pi i} \oint \sum_{j \in \mathbf{Z}}\left(\begin{array}{c}
2 k-2-j \\
n-j
\end{array}\right) x^{-j} \frac{P_{k}(x, 1)}{x} d x \\
& \quad=\frac{1}{2 \pi i} \oint \sum_{r \geq 0}\left(\begin{array}{c}
2 k-2-n-r \\
r
\end{array}\right) x^{r-n} \frac{P_{k}(x, 1)}{x} d x \\
& =\frac{1}{2 \pi i} \oint \frac{1}{(1-x)^{2 k-1-n}} \frac{P_{k}(x, 1)}{x^{n+1}} d x \\
& =\frac{1}{2 \pi i} \oint \frac{P_{k}(x, 1)}{(x /(1-x))^{1+n}(1-x)^{2 k}} d x \\
& =\frac{1}{2 \pi i} \oint(1+t)^{2 k} \frac{P_{k}(t,(1+t))}{t^{n+1}} d t \\
& =\frac{1}{2 \pi i} \oint \frac{P_{k}(t,(1+t))}{t^{n+1}} d t,
\end{aligned}
$$

which is equal to the coefficient of $t^{n}$ in $P_{k}(t,(1+t) y)$. But

$$
P_{k}(t,(1+t) y)=\left((a+b+c) t^{2}+(b+2 c) t y+c y^{2}\right)^{k-1},
$$

and so this coefficient is precisely $d_{n, k}(a+b+c, b+2 c, c)$.

Corollary.

$$
d_{n, k}(a, b+2 a, a+b+c)=\sum_{j=n}^{2 k-2}\left(\begin{array}{l}
j \\
n
\end{array}\right) d_{j, k}(a, b, c) .
$$

ProOF.

$$
\begin{aligned}
d_{n, k}(a, b+2 a, a+b+c) & =d_{2 k-2-n, k}(a+b+c, b+2 a, a) \\
& =\sum_{j=0}^{2 k-2-n}\left(\begin{array}{c}
2 k-2-j \\
2 k-2-n-j
\end{array}\right) d_{j, k}(c, b, a) \\
& =\sum_{j=0}^{2 k-2-n}\left(\begin{array}{c}
2 k-2-j \\
n
\end{array}\right) d_{2 k-2-j, k}(a, b, c) \\
& =\sum_{j=n}^{2 k-2}\left(\begin{array}{l}
j \\
n
\end{array}\right) d_{j, k}(a, b, c)
\end{aligned}
$$

which was to be demonstrated.

We also observe that

$$
\begin{aligned}
\sum_{n=0}^{2 k-2} \frac{d_{n, k}(c, b, a)}{2 k-1-n} & =\sum_{n=0}^{2 k-2} \frac{d_{2 k-2-n, k}(a, b, c)}{2 k-1-n} \\
& =\sum_{n=0}^{2 k-2} \frac{d_{n, k}(a, b, c)}{n+1} .
\end{aligned}
$$


We now substitute (13), (14), and (15) into (12) as follows:

$$
\sum_{n=0}^{2 k} \frac{-d_{n, k+1}(c,-b, a)}{2 k+1-n}=\sum_{n=0}^{2 k} \frac{-d_{n, k+1}(a,-b, c)}{n+1}
$$

$$
\begin{aligned}
\sum_{n=0}^{2 k} & \frac{d_{n, k+1}(a, b-2 a, c-b+a)}{2 k+1-n} \\
& =\sum_{n=0}^{2 k} \frac{(-1)^{n} d_{n, k+1}(a, 2 a-b, c-b+a)}{2 k+1-n} \\
& =\sum_{n=0}^{2 k} \frac{(-1)^{n}}{2 k+1-n} \sum_{j=n}^{2 k}\left(\begin{array}{l}
j \\
n
\end{array}\right) d_{j, k+1}(a,-b, c) \\
& =\sum_{j=0}^{2 k} \sum_{n=0}^{j} \frac{(-1)^{n}}{2 k+1-n}\left(\begin{array}{l}
j \\
n
\end{array}\right) d_{j, k+1}(a,-b, c),
\end{aligned}
$$

(iii)

$$
\begin{aligned}
& \sum_{n=0}^{2 k} \frac{B_{n+1}}{n+1} \frac{B_{2 k+1-n}}{2 k+1-n} d_{n, k+1}(c, 2 c-b, c) \\
& \quad=\sum_{n=0}^{2 k} \frac{B_{2 k+1-n}}{2 k+1-n} \frac{B_{n+1}}{n+1} d_{n, k+1}(a-b+c, 2 c-b, c) \\
& \quad=\sum_{n=0}^{2 k} \frac{B_{n+1}}{n+1} \frac{B_{2 k+1-n}}{2 k+1-n} \sum_{j=0}^{n}\left(\begin{array}{c}
2 k-j \\
n-j
\end{array}\right) d_{j, k+1}(a,-b, c) \\
& \quad=\sum_{j=0}^{2 k} \sum_{n=0}^{2 k-j} \frac{B_{n+1}}{n+1} \frac{B_{2 k+1-n}}{2 k+1-n}\left(\begin{array}{c}
2 k-j \\
n
\end{array}\right) d_{j, k+1}(a,-b, c)
\end{aligned}
$$

Collecting like terms, we obtain

$$
J_{k}(Q)=\sum_{n=0}^{2 k} G_{n, 2 k} \cdot d_{n, k+1}(a,-b, c),
$$

where for $k \geq 1,0 \leq n \leq k$

$$
\begin{aligned}
G_{n, k}= & \frac{B_{k+2}}{k+2} \sum_{j=0}^{k} \frac{(-1)^{j}}{k+1-j}\left(\begin{array}{c}
n \\
j
\end{array}\right)+\sum_{j=0}^{n-1}\left(\begin{array}{l}
n \\
j
\end{array}\right) \frac{B_{j+1}}{j+1} \frac{B_{k+1-j}}{k+1-j} \\
& +\sum_{j=0}^{k-n}\left(\begin{array}{c}
k-n \\
j
\end{array}\right) \frac{B_{j+1}}{j+1} \frac{B_{k+1-j}}{k+1-j} .
\end{aligned}
$$

We shall now show that $G_{n, k}=0$ for even, positive $k$. We shall actually show more; namely, that $G_{n, k}=-B_{k+1} /(k+1)$ for all $k \geq 0,0 \leq n \leq k$.

To this end we introduce the generating function

$$
G(X, Y)=\sum_{\substack{k \geq 0 \\ 0 \leq n \leq k}} G_{n, k} \frac{X^{k-n}}{(k-n) !} \frac{Y^{n}}{n !} .
$$


We first observe that

$$
\begin{aligned}
(-1)^{n} \sum_{j=0}^{n} \frac{(-1)^{j}}{k-j-1}\left(\begin{array}{c}
n \\
j
\end{array}\right) & =\sum_{j=0}^{n}(-1)^{n-j}\left(\begin{array}{c}
k \\
j
\end{array}\right) \int_{0}^{1} t^{k-j} d t \\
& =\int_{0}^{1} t^{k-n}(1-t)^{n} d t=\frac{(k-n) ! n !}{(k+1) !}
\end{aligned}
$$

Hence

$$
\begin{aligned}
& G(X, Y)=\sum_{\substack{k \geq 0 \\
0 \leq n \leq k}}(-1)^{n} \frac{B_{k+2}}{(k+2) !} X^{k-n} Y^{n} \\
& +\sum_{0 \leq n \leq k} \sum_{j=0}^{n-1} \frac{B_{j+1}}{(j+1) !} \frac{B_{k+1-j}}{(k+1-j) !}\left(\begin{array}{c}
k-j \\
k-n
\end{array}\right) X^{k-n} Y^{n} \\
& +\sum_{0 \leq n \leq k} \sum_{j=0}^{k-n} \frac{B_{j+1}}{(j+1) !} \frac{B_{k+1-j}}{(k+1-j) !}\left(\begin{array}{c}
k-j \\
n
\end{array}\right) X^{k-n} Y^{n} \\
& +\sum_{0 \leq n \leq k} \frac{B_{k+2}}{(k+2) !}\left(\begin{array}{c}
k+2 \\
n+1
\end{array}\right) X^{k-n} Y^{n} \\
& =\sum_{k \geq 0} \frac{B_{k+2}}{(k+2) !} \frac{1-(-Y / X)^{k+1}}{1+Y / X} X^{k} \\
& +\sum_{j=0}^{\infty} \sum_{\nu \geq s \geq 0}\left(\frac{B_{j+1}}{(j+1) !} Y^{j} \frac{B_{\nu+1}}{(\nu+1) !} X^{\nu}\left(\begin{array}{l}
\nu \\
s
\end{array}\right)\left(\frac{Y}{X}\right)^{s}\right. \\
& +\sum_{\substack{0 \leq j \\
0 \leq \nu}} \frac{B_{j+1}}{(j+1) !} \frac{B_{\nu+1}}{(\nu+1) !} X^{j+\nu}\left(\frac{X+Y}{X}\right)^{\nu} \\
& \left.+\left(\frac{X}{Y}\right) \sum_{k \geq 0} \frac{B_{k+2}}{(k+2) !} X^{k}\left(\left(\frac{X+Y}{X}\right)^{(k+2)}-1-\left(\frac{Y}{X}\right)^{k+2}\right)\right) \\
& =\frac{1}{X+Y}\left(\frac{1}{e^{X}-1}-\frac{1}{X}-\left(\frac{1}{e^{-Y}-1}+\frac{1}{Y}\right)\right)+\sum_{j=0}^{\infty} \frac{B_{j+1}}{(j+1) !} Y^{j} \\
& \cdot \sum_{\nu=0}^{\infty} \frac{B_{\nu+1}}{(\nu+1) !} X^{\nu}\left(\left(\frac{X+Y}{X}\right)^{\nu}-1\right) \\
& +\left(\frac{1}{e^{X}-1}-\frac{1}{X}\right)\left(\frac{1}{e^{X+Y}-1}-\frac{1}{X+Y}\right) \\
& +\frac{1}{Y}\left(\frac{X+Y}{X}\left(\frac{1}{e^{X+Y}-1}-\frac{1}{X+Y}\right)\right. \\
& \left.-\left(\frac{1}{e^{X}-1}-\frac{1}{X}\right)-Y\left(\frac{1}{e^{Y}-1}-\frac{1}{Y}\right)\right)
\end{aligned}
$$




$$
\begin{aligned}
= & \left(\frac{1}{X+Y}\left(\frac{1}{e^{X}-1}-\frac{1}{e^{-Y}-1}\right)-\frac{1}{X Y}\right) \\
& +\left(\frac{1}{e^{Y}-1}-\frac{1}{Y}\right)\left(\frac{1}{e^{X+Y}-1}-\frac{1}{X+Y}-\frac{1}{e^{X}-1}+\frac{1}{X}\right) \\
& +\left(\frac{1}{e^{X}-1}-\frac{1}{X}\right)\left(\frac{1}{e^{X+Y}-1}-\frac{1}{X+Y}\right) \\
& +\frac{1}{Y}\left(\frac{X+Y}{X}\left(\frac{1}{e^{X+Y}-1}-\frac{1}{X+Y}\right)\left(\frac{1}{e^{X}-1}-\frac{1}{X}\right)-Y\left(\frac{1}{e^{Y}-1}-\frac{1}{Y}\right)\right) \\
= & \frac{1}{X+Y}-\frac{1}{e^{X+Y}-1}=-\sum_{k=0}^{\infty} \frac{B_{k+1}}{(k+1) !}(X+Y)^{k} .
\end{aligned}
$$

Comparing this with (16), we see indeed that $G_{n, k}=-B_{k+1} /(k+1)$, as was to be shown.

Proof OF THEOREM 5. To show $Z_{k}(Q) \stackrel{(-1)^{k}}{\sim} 0$ we must find functions $F_{k}(Q)$ satisfying

(a) $F_{k}\left(w, w^{\prime}\right)=(-1)^{k} F_{k}\left(1 / w, 1 / w^{\prime}\right) ; k=1,2,3, \ldots$,

(b) $F_{k}\left(w-1,1-w^{\prime}\right)-F_{k}\left(1-1 / w, 1 / w^{\prime}-1\right)=Z_{k}(Q) ; k=1,2,3, \ldots$. Let

$$
\begin{aligned}
S_{k}(Q)=D^{k / 2} \frac{k !}{2}\left(w+w^{\prime}\right)^{-k} \sum_{r=0}^{2 k} & \frac{B_{r+1}}{(r+1) !} \frac{B_{2 k+1-r}}{(2 k+1-r) !} \\
& \cdot\left(\frac{\partial^{k}}{\partial w^{k}} \frac{w^{r}}{\left(w+w^{\prime}\right)^{k+1}}-\frac{\partial^{k}}{\partial w^{\prime k}} \frac{w^{\prime r}}{\left(w+w^{\prime}\right)^{k+1}}\right) .
\end{aligned}
$$

We then assert that the functions

$$
\begin{aligned}
F_{k}(Q)=D^{k / 2} \frac{(-1)^{k} k !}{2} \frac{B_{2 k+2}}{2 k+2}( & \left(w+w^{\prime}\right)^{-k} f_{k}\left(w, w-w^{\prime},-w^{\prime}\right) \\
& \left.+(-1)^{k}\left(w w^{\prime}\right)^{k} f_{k}\left(\frac{1}{w}, \frac{w^{\prime}-w}{w w^{\prime}}, \frac{-1}{w^{\prime}}\right)\right)-S_{k}\left(w, w^{\prime}\right)
\end{aligned}
$$

have the desired properties.

It is easily checked that (a) is satisfied. To establish (b), we begin with the following lemma.

LEMMA 3. For $k \leq 0,0 \leq n \leq 2 k$, let

$$
\Delta_{n, k}=\Delta_{n, k}(a, b, c)=\frac{(-1)^{k}}{2}\left(\begin{array}{c}
2 k \\
n
\end{array}\right)\left(w-w^{\prime}\right)^{k+1} \frac{\partial^{2 k}}{\partial w^{k} \partial w^{\prime k}}\left(\frac{w^{n}+w^{\prime n}}{w-w^{\prime}}\right) .
$$

Then $\left(D^{k / 2} /(2 k) !\right) \Delta_{n, k}(a, b, c)=d_{n, k}(c, b, a)$.

ProOF. Using Leibniz' formula for the derivative, we obtain

$$
\begin{aligned}
\Delta_{n, k}= & \frac{(-1)^{k}\left(w-w^{\prime}\right)^{k+1}}{2}\left(\begin{array}{c}
2 k \\
n
\end{array}\right) \sum_{r=0}^{2 k}\left(\begin{array}{c}
k \\
r
\end{array}\right) \frac{n !}{(n-r) !}(-1)^{k}(2 k-r) ! \\
& \cdot\left(w-w^{\prime}\right)^{r-(2 k+1)}\left((-1)^{r} w^{n-r}+w^{n-r}\right) \\
= & (2 k) !\left(w-w^{\prime}\right)^{-k} \sum_{r=0}^{2 k}\left(\begin{array}{c}
k \\
r
\end{array}\right)\left(\begin{array}{c}
2 k-r \\
n-r
\end{array}\right)\left(w-w^{\prime}\right)^{r}\left((-1)^{r} w^{n-r}+w^{\prime n-r}\right) .
\end{aligned}
$$


We now introduce the generating function

$$
\begin{aligned}
& \Delta=\frac{1}{(2 k) !} \sum_{0 \leq n \leq 2 k} \Delta_{n, k} Y^{k} \\
& =\frac{1}{2}\left(w, w^{\prime}\right)^{-k} \sum_{r=0}^{2 k} Y^{r}\left(\begin{array}{l}
k \\
r
\end{array}\right)\left(w-w^{\prime}\right)^{r} \\
& \left((-1)^{r} \sum_{n=r}^{2 k}\left(\begin{array}{c}
2 k-r \\
n-r
\end{array}\right)(w Y)^{n-r}+\sum_{n=r}^{2 k}\left(\begin{array}{c}
2 k-r \\
n-r
\end{array}\right)\left(w^{\prime} Y\right)^{n-r}\right) \\
& =\frac{1}{2}\left(w-w^{\prime}\right)^{-k} \sum_{r \in \mathbf{Z}}\left(\begin{array}{l}
k \\
r
\end{array}\right)\left(Y\left(w-w^{\prime}\right)\right)^{r}\left((-1)^{r}(1+w Y)^{2 k-r}+\left(1+w^{\prime} Y\right)^{2 k-r}\right) \\
& =\frac{1}{2}\left(w-w^{\prime}\right)^{k} \operatorname{Tr}\left(\left(1+w^{\prime} Y\right)^{k}(1+w Y)^{k}\right)=D^{-k / 2} a^{k}\left(1+\frac{b}{a} Y+\frac{c}{a} Y^{2}\right)^{k} \\
& =D^{-k / 2}\left(a+b Y+c Y^{2}\right)^{k} \text {. }
\end{aligned}
$$

Thus

$$
\frac{1}{(2 k) !} \sum_{0 \leq n \leq 2 k} \Delta_{n, k} Y^{n}=D^{-k / 2}\left(a+b Y+c Y^{2}\right)^{k} .
$$

Comparing coefficients of $Y^{n}$, we see that

$$
\frac{D^{k / 2}}{(2 k) !} \Delta_{n, k}(a, b, c)=d_{k, n}(c, b, a)
$$

as was to be shown.

Applying the lemma to $S_{k}$, and recalling that if the pair $\left(w, w^{\prime}\right)$ corresponds to the form $(a, b, c)$, then $\left(w-1, w^{\prime}-1\right)$ corresponds to $(a, b-2 a, a-b+c)$ and $\left(1-1 / w, 1-1 / w^{\prime}\right)$ to $(c, 2 c-b, c-b+a)$, we obtain

$S_{k}\left(w-1,1-w^{\prime}\right)$

$$
\begin{aligned}
=D^{k / 2} \frac{k !}{2}\left(w-w^{\prime}\right)^{k+1} & \sum_{r=0}^{2 k} \frac{B_{r+1}}{(r+1) !} \frac{B_{2 k+1-r}}{(2 k+1-r) !} \\
& \cdot\left(\frac{\partial^{k}}{\partial w^{k}} \frac{(w-1)^{r}}{\left(w-w^{\prime}\right)^{k+1}}+(-1)^{r} \frac{\partial^{k}}{\partial w^{\prime k}} \frac{(w-1)^{r}}{\left(w-w^{\prime}\right)^{k+1}}\right) \\
=D^{k / 2} \frac{1}{2}\left(w-w^{\prime}\right)^{k+1} \sum_{r=0}^{2 k} \frac{B_{r+1}}{(r+1) !} \frac{B_{2 k+1-r}}{(2 k+1-r) !} \frac{\partial^{2 k}}{\partial w^{k} \partial w^{\prime k}} & \cdot\left(\frac{(w-1)^{r}+\left(w^{\prime}-1\right)^{r}}{w-w^{\prime}}\right)
\end{aligned}
$$

(note: for $k \geq 1$ the terms for even $r$ vanish)

$$
\begin{aligned}
& =\frac{D^{k / 2}}{(2 k) !} \sum_{r=0}^{2 k} \frac{B_{r+1}}{r+1} \frac{B_{2 k+1-r}}{(2 k+1-r) !} \Delta_{n, k}(a, b-2 a, a-b+c) \\
& =-\sum_{r=0}^{2 k} \frac{B_{r+1}}{r+1} \frac{B_{2 k+1-r}}{2 k+1-r} d_{n, k+1}(a, 2 a-b, a-b+c) \\
& =g_{k}(a-b+c, 2 a-b, a)=g_{k}(a, 2 a-b, a-b+c) .
\end{aligned}
$$


Similarly,

$$
S_{k}\left(1-1 / w, 1 / w^{\prime}-1\right)=-g_{k}(c, 2 c-b, c-b+a) .
$$

Proceeding as in the proof of Theorem 4, we obtain

$$
\begin{aligned}
F_{k}(w- & \left.1,1-w^{\prime}\right)-F_{k}\left(1-\frac{1}{w}, \frac{1}{w^{\prime}}-1\right)-Z_{k}(Q) \\
= & \frac{(-1)^{k} k !}{2} \frac{B_{2 k+2}}{2 k+2}\left(\frac{\varphi_{k}(D, b-2 a)-\varphi_{k}(D, b)}{a^{k+1}}+\frac{\varphi_{k}(D, b-2 c)-\varphi_{k}(D, b)}{c^{k+1}}-\frac{\varphi_{k}(D, 2 a-b)-\varphi_{k}(D, b-2 c)}{(c-b+a)^{k+1}}\right) \\
& +g_{k}(a, 2 a-b, a-b+c)+g_{k}(c, 2 c-b, c-b+a)-g_{k}(a,-b, c) \\
= & -\frac{B_{2 k+2}}{2 k+2} \sum_{n=0}^{2 k}\left(\frac{d_{n, k+1}(c,-b, a)}{2 k+1-n}+\frac{d_{n, k+1}(a,-b, c)}{2 k+1-n}\right. \\
& +g_{k}(a, 2 a-b, a-b+c)+g_{k}(c, 2 c-b, c-b+a)-g_{k}(a,-b, c) \\
= & -J_{k}(Q)-2 \frac{B_{2 k+2}}{2 k+2} \sum_{n=0}^{2 k}\left(\frac{d_{n, k+1}(c,-b, a)}{2 k+1-n}+\frac{d_{n, k+1}(a,-b, c)}{2 k+1-n}\right) \\
& +2 B_{2 k+2} \sum_{n=0}^{k} \frac{1}{(n+1)(2 k+1-n)} d_{n, k+1}(a,-b, c) .
\end{aligned}
$$

But this equals zero, since $J_{k}(Q)=0$, and by (15)

$$
\begin{aligned}
\frac{1}{2 k+2} & \sum_{n=0}^{2 k}\left(\frac{d_{n, k+1}(c,-b, a)}{2 k+1-n}+\frac{d_{n, k+1}(a,-b, c)}{2 k+1-n}\right) \\
= & \frac{1}{2 k+2} \sum_{n=0}^{2 k}\left(\frac{d_{n, k+1}(a,-b, c)}{n+1}+\frac{d_{n, k+1}(a,-b, c)}{2 k+1-n}\right) \\
= & \sum_{n=0}^{2 k} \frac{1}{(n+1)(2 k+1-n)} d_{n, k+1}(a,-b, c) .
\end{aligned}
$$

Thus we have shown that indeed

$$
F_{k}\left(w-1,1-w^{\prime}\right)-F_{k}\left(1-1 / w, 1 / w^{\prime}-1\right)=Z_{k}(Q) .
$$

For the proof of Theorem 6 we turn to [2], in which Shanks and Zagier give a formula for the value of $Z_{Q}(s)$ at positive integers. Here we use a slightly modified definition of $Z_{Q}$ :

$$
Z_{Q}(s)=\sum_{p=1}^{\infty} \sum_{q=0}^{\infty} \frac{1}{Q(p, q)^{s}} ; \quad \operatorname{re}(s)>1 .
$$

We also define functions

$$
\mathcal{F}_{k}(x)=\sum_{n=1}^{\infty} \frac{\psi(n x)}{n^{2 k+1}} ; \quad k \geq 1, k \in \mathbf{Z}, x>0
$$


where

$$
\psi(x)=\frac{\Gamma^{\prime}(x)}{\Gamma(x)}=\lim _{N \rightarrow \infty}-\sum_{q=0}^{N}\left(\frac{1}{x+q}+\log N\right) .
$$

THEOREM 7 (SHANKS AND ZAGIER). Let $Q$ be a binary quadratic form of discriminant $D>0$ with positive coefficients and roots $w, w^{\prime}$. Then for $k \geq 1, k \in \mathbf{Z}$ (20)

$$
D^{(k+1) / 2} Z_{Q}(1+k)=\sum_{r=0}^{k} \frac{(-1)^{r}}{(k-r) !}\left(\begin{array}{c}
k+r \\
r
\end{array}\right) \frac{\mathcal{F}_{k}^{(k-r)}(w)+(-1)^{k+1-r} \mathcal{F}_{k}^{(k-r)}\left(w^{\prime}\right)}{\left(w-w^{\prime}\right) r},
$$

or equivalently,

$\left(20^{\prime}\right)$

$$
D^{(k+1) / 2} Z_{Q}(1+k)=\frac{\left(w-w^{\prime}\right)^{k+1}}{k !}\left(\frac{\partial^{k}}{\partial w^{k}} \frac{\mathcal{F}_{k}(w)}{\left(w-w^{\prime}\right)^{k+1}}+\frac{\partial^{k}}{\partial w^{\prime k}} \frac{\mathcal{F}_{k}\left(w^{\prime}\right)}{\left(w-w^{\prime}\right)^{k+1}}\right) .
$$

Shanks and Zagier also obtain asymptotic expansions for the $\mathcal{F}_{k}(x)$, and show that they satisfy the following functional equations:

$$
\begin{aligned}
\mathcal{F}_{k}(x)+x^{2 k} \mathcal{F}_{k}\left(\frac{1}{x}\right)= & -\varsigma(2 k+2)\left(x^{-1}+x^{2 k+1}\right)-\gamma \zeta(2 k+1)\left(1+x^{2 k}\right) \\
& +\sum_{r=2}^{2 k}(-1)^{r} \varsigma(r) \varsigma(2 k+2-r) x^{r-1},
\end{aligned}
$$

where $\varsigma(s)$ denotes Riemann's zeta function, and $\gamma$ Euler's constant. For notational convenience later, we define

$$
h_{k}(x)=-\varsigma(2 k+2)\left(x^{-1}+x^{2 k+1}\right),
$$

and $\alpha_{k, r}$ to be the coefficient of $x^{r}, 2 \leq r \leq 2 k$, in the right-hand side of (21). Then (21) becomes

$$
\mathcal{F}_{k}(x)+x^{2 k} \mathcal{F}_{k}\left(\frac{1}{x}\right)=h_{k}(x)+\sum_{r=0}^{2 k} \alpha_{k, r} x^{r}=: A_{k}(x) .
$$

The second functional equation for the $\mathcal{F}_{k}$ proved by Shanks and Zagier is

$$
\mathcal{F}_{k}(x)-\mathcal{F}_{k}(x-1)+x^{2 k} \mathcal{F}_{k}\left(\frac{x-1}{x}\right)=\sum_{j=0}^{2 k} \beta_{k, j} x^{j},
$$

where the $\beta_{k, j}$ are certain constants satisfying the relations

$$
\begin{aligned}
\beta_{k, j}+\beta_{k, 2 k-j}=(-1)^{j} \alpha_{k, j}-\varsigma(2 k+2) ; \quad 0 \leq j \leq 2 k \\
(-1)^{j} \beta_{k, j}+\sum_{n=j}^{2 k}\left(\begin{array}{c}
k \\
j
\end{array}\right) \beta_{k, n}=\left((-1)^{j-1}-\left(\begin{array}{c}
2 k+1 \\
j+1
\end{array}\right)\right) \varsigma(2 k+2) \\
+\sum_{n=0}^{j}\left(\begin{array}{c}
2 k-n \\
j-n
\end{array}\right) \alpha_{k, n} ; \quad 0 \leq j \leq 2 k
\end{aligned}
$$


We then define the polynomial $C_{k}(x)=\sum_{j=0}^{2 k} \beta_{k, j} x^{j}$. Lastly, we define $G_{k}(x)=$ $\mathcal{F}_{k}(x)-\frac{1}{2} A_{k}(x)$ and observe that

$$
\begin{gathered}
G_{k}(x)+x^{2 k} G_{k}\left(\frac{1}{x}\right)=0 \\
G_{k}(x)-G_{k}(x-1)+x^{2 k} G_{k}\left(1-\frac{1}{x}\right) \\
=C_{k}(x)-\frac{1}{2} A_{k}(x)+\frac{1}{2} A_{k}(x-1)-\frac{1}{2} x^{2 k} A_{k}\left(1-\frac{1}{x}\right)=: D_{k}(x) .
\end{gathered}
$$

Now let

$$
R_{k}(x, y)=\frac{(x+y)^{k+1}}{k !}\left(\frac{\partial^{k}}{\partial x^{k}} \frac{G_{k}(x)+\frac{1}{2} h_{k}(x)}{(x+y)^{k+1}}-\frac{\partial^{k}}{\partial y^{k}} \frac{G_{k}(y)-\frac{1}{2} h_{k}(y)}{(x+y)^{k+1}}\right) .
$$

To prove our theorem we shall show that

$$
R_{k}(x, y)=(-1)^{k+1} R_{k}(1 / x, 1 / y)
$$

as well as that

$$
R_{k}\left(w-1,1-w^{\prime}\right)-R_{k}\left(1-1 / w, 1 / w^{\prime}-1\right)=D^{(k+1) / 2} Z_{Q}(1+k)+\delta_{k} K_{k}(Q) .
$$

The first assertion follows easily from (25) and the identity

$$
\frac{d^{k}}{d(1 / z)^{k}} z^{1-k} f(z)=(-1)^{k} z^{k+1} \frac{d^{k}}{d z^{k}} f(z) \text {. }
$$

We now prove the second assertion.

$$
\begin{aligned}
& R_{k}\left(w-1,1-w^{\prime}\right)-R_{k}\left(1-\frac{1}{w}, \frac{1}{w^{\prime}}-1\right) \\
&=\frac{\left(w-w^{\prime}\right)^{k+1}}{k !} \frac{\partial^{k}}{\partial(w-1)^{k}} \frac{G_{k}(w-1)+\frac{1}{2} h_{k}(w-1)}{\left(w-w^{\prime}\right)^{k+1}} \\
& \quad-\frac{\left(w-w^{\prime}\right)^{k+1}}{k !} \frac{\partial^{k}}{\partial\left(1-w^{\prime}\right)^{k}} \frac{G_{k}\left(1-w^{\prime}\right)-\frac{1}{2} h_{k}\left(1-w^{\prime}\right)}{\left(w-w^{\prime}\right)^{k+1}} \\
& \quad-\frac{1}{k !}\left(\frac{w-w^{\prime}}{w w^{\prime}}\right)^{k+1} \frac{\partial^{k}}{\partial(1-1 / w)^{k}} \frac{G_{k}(1-1 / w)+\frac{1}{2} h_{k}(1-1 / w)}{\left(\left(w-w^{\prime}\right) / w w^{\prime}\right)^{k+1}} \\
&+\frac{1}{k !}\left(\frac{w-w^{\prime}}{w w^{\prime}}\right)^{k+1} \frac{\partial^{k}}{\partial\left(1 / w^{\prime}-1\right)^{k}} \frac{G_{k}\left(1-1 / w^{\prime}\right)+\frac{1}{2} h_{k}\left(1-1 / w^{\prime}\right)}{\left(\left(w-w^{\prime}\right) / w w^{\prime}\right)^{k+1}} \\
&=\frac{\left(w-w^{\prime}\right)^{k+1}}{k !} \frac{\partial^{k}}{\partial w^{k}} \frac{G_{k}(w)-D_{k}(w)+\frac{1}{2} \zeta(2 k+2)\left(\left(w^{2 k+1}-1\right) /(w-1)-(w-1)^{2 k+2} / w\right)}{\left(w-w^{\prime}\right)^{k+1}} \\
&\left.+\frac{(-1)^{k+1}\left(w-w^{\prime}\right)^{k+1}}{k !} \frac{\partial^{k}}{\partial w^{\prime k}} \quad-\frac{D_{k}(w)+w^{2 k} D_{k}\left(1 / w^{\prime}\right)}{w-w^{\prime}}\right) \\
& \quad \frac{G_{k}\left(w^{\prime}\right)+w^{\prime 2 k} D_{k}\left(1 / w^{\prime}\right)+\frac{1}{2} \varsigma(2 k+2)\left(\left(1-w^{\prime}\right)^{2 k+1} /\left(1-w^{\prime}\right)-\left(1-w^{\prime}\right)^{2 k+2} / w^{\prime}\right)}{\left(w-w^{\prime}\right)^{k+1}} \\
&=\frac{\left(w-w^{\prime}\right)^{k+1}}{(k !)^{2}} \frac{\partial^{2 k}}{\partial w^{k} \partial w^{\prime k}}\left(\frac{G_{k}(w)-\frac{1}{2} \varsigma(2 k)\left(1 / w+w^{2 k+1}\right)-G_{k}\left(w^{\prime}\right)+\frac{1}{2}(2 k)\left(1 / w^{\prime}+w^{\prime 2 k+1}\right)}{w-w^{\prime}}\right. \\
&= D^{(k+1) / 2} \cdot Z_{Q}(1+k)-\frac{\left(w-w^{\prime}\right)^{k+1}}{(k !)^{2}} \frac{\partial^{2 k}}{\partial w^{k} \partial w^{\prime k}} \frac{D_{k}(w)+w^{\prime 2 k} D_{k}\left(1 / w^{\prime}\right)}{w-w^{\prime}} .
\end{aligned}
$$


In the last two steps we made use of the fact that if $P(X)$ is a polynomial of degree at most $2 k$, then

$$
\frac{\partial^{2 k}}{\partial w^{k} \partial w^{\prime k}} \frac{P(w)-P\left(w^{\prime}\right)}{w-w^{\prime}}=0 .
$$

It remains then to be shown that

$$
\frac{\left(w-w^{\prime}\right)^{k+1}}{(k !)^{2}} \frac{\partial^{2 k}}{\partial w^{k} \partial w^{\prime k}} \frac{D_{k}(w)+w^{\prime 2 k} D_{k}\left(1 / w^{\prime}\right)}{w-w^{\prime}}=D^{(k+1) / 2} \cdot \delta_{k} K_{k}(a, b, c) .
$$

Now

$$
\begin{aligned}
& D_{k}(x)=C_{k}(x)=\frac{1}{2} A_{k}(x)+\frac{1}{2} A_{k}(x-1)-\frac{1}{2} x^{2 k} A_{k}(1-1 / x) \\
& =\sum_{j=0}^{2 k} \beta_{k, j} x^{j}+\frac{1}{2} \zeta(2 k+2)\left(\frac{1}{x}+x^{2 k+1}\right) \\
& -\frac{1}{2} \sum_{j=0}^{2 k} \alpha_{k, j} x^{j}-\frac{1}{2} \zeta(2 k+2)\left(\frac{1}{x-1}+(x-1)^{2 k+1}\right) \\
& +\frac{1}{2} \sum_{j=0}^{2 k} \alpha_{k, j}(x-1)^{j}+\frac{1}{2} \zeta(2 k+2)\left(\frac{x^{2 k-1}}{x-1}+\frac{(x-1)^{2 k+1}}{x}\right) \\
& -\sum_{j=0}^{2 k} \alpha_{k, j} x^{2 k-j}(x-1)^{j} \\
& =\sum_{n=0}^{2 k}\left(\beta_{k, n}+\frac{1}{2} \varsigma(2 k)+(-1)^{n} \frac{1}{2}\left(\begin{array}{c}
2 k+2 \\
n+1
\end{array}\right) \varsigma(2 k+2)\right. \\
& +(-1)^{n} \frac{1}{2}\left(\begin{array}{c}
2 k+1 \\
n
\end{array}\right) \varsigma(2 k+2) \\
& -\frac{1}{2} \alpha_{k, n}+(-1)^{n} \frac{1}{2} \sum_{j=n}^{2 k}(-1)^{j} \alpha_{k, j}\left(\begin{array}{l}
j \\
n
\end{array}\right) \\
& \left.-(-1)^{n} \sum_{j=2 k-n}^{2 k} \alpha_{k, j}\left(\begin{array}{c}
j \\
2 k-n
\end{array}\right)\right) x^{n} \\
& =\sum_{n=0}^{2 k}\left(\beta_{k, n}+\frac{1}{2} \varsigma(2 k+2)+(-1)^{n} \frac{1}{2}\left(\begin{array}{c}
2 k+2 \\
n+1
\end{array}\right) \varsigma(2 k+2)-\frac{1}{2} \alpha_{k, n}\right. \\
& +\frac{(-1)^{n}}{2} \sum_{\substack{j=0 \\
j \text { even }}}^{2 k} \alpha_{k, j}\left(\left(\begin{array}{l}
j \\
n
\end{array}\right)-\left(\begin{array}{c}
j \\
2 k-n
\end{array}\right)\right) \\
& \left.-\frac{(-1)^{n}}{2} \sum_{\substack{j=0 \\
j \text { odd }}}^{2 k} \alpha_{k, j}\left(\left(\begin{array}{l}
j \\
n
\end{array}\right)+\left(\begin{array}{c}
j \\
2 k-n
\end{array}\right)\right)\right) x^{n} .
\end{aligned}
$$


We now proceed to compute $x^{2 k} D_{k}(1 / x)$. Using relation (23) we obtain

$$
\begin{aligned}
& x^{2 k} D_{k}\left(\frac{1}{x}\right)=\sum_{n=0}^{2 k}\left(-\beta_{k, n}+(-1)^{n} \alpha_{k, n}-\frac{1}{2} \varsigma(2 k+2)+\frac{(-1)^{n}}{2}\left(\begin{array}{c}
2 k+2 \\
k+1
\end{array}\right) \varsigma(2 k+2)\right. \\
& -\frac{1}{2} \alpha_{k, n}-\frac{(-1)^{n}}{2} \sum_{\substack{j=0 \\
j \text { even }}}^{2 k} \alpha_{k, j}\left(\left(\begin{array}{l}
j \\
n
\end{array}\right)-\left(\begin{array}{c}
j \\
2 k-n
\end{array}\right)\right) \\
& \left.-\frac{(-1)^{n}}{2} \sum_{\substack{j=0 \\
j \text { odd }}}^{2 k} \alpha_{k, j}\left(\left(\begin{array}{l}
j \\
k
\end{array}\right)+\left(\begin{array}{c}
j \\
2 k-n
\end{array}\right)\right)\right) x^{n}
\end{aligned}
$$

We have, therefore,

$\frac{\left(w-w^{\prime}\right)^{k+1}}{(k !)^{2}} \frac{\partial^{2 k}}{\partial w^{k} \partial w^{\prime k}} \frac{D_{k}(w)+w^{2 k} D_{k}\left(1 / w^{\prime}\right)}{w-w^{\prime}}=\frac{\left(w-w^{\prime}\right)^{k+1}}{(k !)^{2}} \frac{\partial^{2 k}}{\partial w^{k} \partial w^{\prime k}}$

$$
\times \sum_{k=0}^{2 k} \frac{1}{w-w^{\prime}}\left(\left(\beta_{k, n}+\frac{1}{2} \zeta(2 k+2)\right.\right.
$$

$$
\begin{aligned}
& \left.+\frac{(-1)^{n}}{2} \sum_{\substack{j=0 \\
j \text { even }}}^{2 k} \alpha_{k, j}\left(\left(\begin{array}{l}
j \\
n
\end{array}\right)-\left(\begin{array}{c}
j \\
2 k-n
\end{array}\right)\right)\right)\left(w^{n}-w^{\prime n}\right) \\
& +\left(-\frac{1}{2} \alpha_{k, n}+\frac{(-1)^{n}}{2}\left(\begin{array}{c}
2 k+2 \\
n+1
\end{array}\right) \varsigma(2 k+2)\right. \\
& \left.-\frac{(-1)^{n}}{2} \sum_{\substack{j=0 \\
j \text { odd }}}^{2 k} \alpha_{k, j}\left(\left(\begin{array}{l}
j \\
n
\end{array}\right)+\left(\begin{array}{c}
j \\
2 k-n
\end{array}\right)\right)\right) \\
& \left.\times\left(w^{n}+w^{\prime n}\right)+(-1)^{n} \alpha_{k, n} w^{\prime n}\right) .
\end{aligned}
$$

By remark (27) the coefficient of $\left(w^{n}-w^{\prime n}\right)$ contributes zero to this expression, and, moreover, we may replace $(-1)^{n} \alpha_{k, n} w^{\prime n}$ by

$$
(-1)^{n} \alpha_{k, n} w^{\prime n}+(-1)^{n} \frac{1}{2} \alpha_{k, n}\left(w^{n}-w^{\prime n}\right)=\frac{(-1)^{n}}{2} \alpha_{k, n}\left(w^{n}+w^{\prime n}\right),
$$

and we see that the right-hand side of (28) equals

$$
\begin{aligned}
& \sum_{n=0}^{2 k} \frac{(-1)^{n}}{2}\left(\begin{array}{c}
2 k+2 \\
n+1
\end{array}\right) \varsigma(2 k+2)-\frac{(-1)^{n}}{2} \sum_{\substack{j=0 \\
j \text { odd }}}^{2 k} \alpha_{k, j}\left(\left(\begin{array}{c}
j \\
n
\end{array}\right)+\left(\begin{array}{c}
j \\
2 k-n
\end{array}\right)\right) \\
& +\frac{1}{2}\left(-1+(-1)^{n}\right) \alpha_{k, n} \frac{\left(w-w^{\prime}\right)^{k+1}}{(k !)^{2}} \frac{\partial^{2 k}}{\partial w^{k} \partial w^{r^{k}}} \frac{w^{n}+w^{\prime n}}{w-w^{\prime}} .
\end{aligned}
$$


But by Lemma 3

$$
\frac{\left(w-w^{\prime}\right)^{k+1}}{(k !)^{2}} \frac{\partial^{2 k}}{\partial w^{k} \partial w^{\prime k}} \frac{w^{n}+w^{\prime n}}{w-w^{\prime}}=(-1)^{k+n} 2 D^{-k / 2} \frac{\left(\begin{array}{c}
2 k \\
k
\end{array}\right)}{\left(\begin{array}{c}
2 k \\
n
\end{array}\right)} d_{n, k+1}(c, b, a)
$$

and so we have

$$
\begin{aligned}
& \frac{\left(w-w^{\prime}\right)^{k+1}}{(k !)^{2}} \frac{\partial^{2 k}}{\partial w^{k} \partial w^{\prime k}} \frac{D_{k}(w)+w^{2 k} D_{k}\left(1 / w^{\prime}\right)}{w-w^{\prime}} \\
& \begin{aligned}
=(-1)^{k} D^{-k / 2}\left(\begin{array}{c}
2 k \\
k
\end{array}\right) \sum_{n=0}^{2 k}\left(\begin{array}{c}
2 k \\
n
\end{array}\right)^{-1} & \left(\left(\begin{array}{c}
2 k+2 \\
n+1
\end{array}\right) \varsigma(2 k+2)\right. \\
& \left.-\sum_{\substack{j=0 \\
j \text { odd }}}^{2 k} \alpha_{k, j}\left(\left(\begin{array}{c}
j \\
n
\end{array}\right)+\left(\begin{array}{c}
j \\
2 k-n
\end{array}\right)\right)\right) \\
-\left(-1+(-1)^{n}\right) \alpha_{k, n} d_{n, k+1}(c, b, a) . &
\end{aligned}
\end{aligned}
$$

Writing

$$
\varsigma(2 k+2)=(-1)^{k} \frac{B_{2 k+2}}{2 k+2} \frac{2^{2 k+1} \pi^{2 k+2}}{(2 k+1) !},
$$

and observing that for $j$ odd,

$$
\alpha_{k, j}=\varsigma(j+1) \zeta(2 k+1-j)=2^{2 k} \pi^{2 k+2} \frac{B_{j+1}}{(j+1) !} \frac{B_{2 k+1-j}}{(2 k+1-j) !},
$$

the left side of (29) is seen to be equal to

$$
\begin{aligned}
& \frac{D^{-k / 2} \pi^{2 k+2} 2^{2 k}}{\Gamma^{2}(k+1)}\left(-\sum_{n=0}^{2 k} \frac{2 B_{2 k+2}}{(n+1)(2 k+1-n)} d_{n, k+1}(c, b, a)\right. \\
& +2 \sum_{n=1}^{2 k} \frac{B_{n+1}}{n+1} \frac{B_{2 k+1-n}}{2 k+1-n} d_{n, k+1}(c, b, a) \\
& -\sum_{j=1}^{2 k} \frac{B_{j+1}}{j+1} \frac{B_{2 k+1-j}}{2 k+1-j} \sum_{n=0}^{2 k}\left(\begin{array}{c}
2 k-n \\
j-n
\end{array}\right) \\
& \left.\cdot\left(d_{n, k+1}(a, b, c)+d_{n, k+1}(c, b, a)\right)\right) \text {. }
\end{aligned}
$$

But by Lemma 2

$$
\begin{aligned}
& \sum_{n=0}^{2 k}\left(\begin{array}{c}
2 k-n \\
j-n
\end{array}\right)\left(d_{n, k+1}(a, b, c)+d_{n, k+1}(c, b, a)\right) \\
& \quad=d_{j, k+1}(a+b+c, b+2 c, c)+d_{j, k+1}(c+b+a, b+2 a, a) .
\end{aligned}
$$


Thus we have shown (at last!) that

$$
\frac{\left(w-w^{\prime}\right)^{k+1}}{(n !)^{2}} \frac{\partial^{2 k}}{\partial w^{k} \partial w^{\prime k}}\left(\frac{D_{k}(w)+w^{2 k} D_{k}\left(1 / w^{\prime}\right)}{w-w^{\prime}}\right)=\frac{D^{-k / 2} \pi^{2 k+2} 2^{2 k}}{\Gamma^{2}(k+1)} K_{k}(a, b, c),
$$

completing the proof of the theorem.

\section{REFERENCES}

1. Z. I. Borevich and I. R. Shafarevich, Number theory, Academic Press, New York, 1966.

2. D. Shanks and D. Zagier, On the values of zeta functions of real quadratic fields at positive integers, in preparation. (Preprint available from Don Zagier, University of Maryland.)

3. D. Zagier, A Kronecker limit formula for real quadratic fields, Math. Ann. 213 (1975), 153-184.

4. __ Valeurs des fonctions zêta des corps quadratiques réels aux entiers négatifs, J. Arithmétiques de Caen, Astérisque 41-42 (1977), 135-151.

5. Takuro Shintani, On evaluation of zeta functions of totally real algebraic number fields on non-positive integers, J. Fac. Sci. Univ. Tokyo 23 (1976), 393-417.

School of Communication and Cognitive Science, Hampshire College, AmHERST, MASSACHUSETTS 01002 